Artikel

Ulrich van Loyen*

\title{
Strich durch die Rechnung. Eine Anmerkung zur Restitutionsdebatte, die Deutschland drei Jahre in Atem hielt.
}


Abstract: Der Artikel setzt sich, durchaus polemisch, mit einigen Motiven des jüngsten Diskurses über die Rückgabe von Artefakten aus deutschen Museen an staatliche Institutionen auf dem Boden ehemaliger Kolonialgebiete auseinander. Er vertritt die These, dass dadurch gerade keine Entschuldigung stattfindet (ganz im Sinne von Marcel Mauss' „reçevoir est reçu“), sondern bestehende regionale Konflikte vertieft und neokolonialistische Verhaltensweisen befördert werden. Zudem plädiert er dafür, die Wissenschaft möge sich nicht voreilig von der Politik entmündigen lassen.

Keywords: Restitution, Kolonialismus, Raubkunst, Cultural Property, Nationalstaaten, Source Communities

*Dr. Dr. Ulrich van Loyen, Universität Siegen, Medienwissenschaftliches Seminar, E-Mail: Ulrich.vLoyen@uni-siegen.de

Eine Schwalbe macht noch keinen Sommer. Nachdem die Kunsthistorikerin Bénedicte Savoy auch hierzulande die populäre Version ihres "Restitutions"-Aufrufs (2018) veröffentlicht und Emmanuel Macron die Rückgabe geraubter Kulturgüter zur obersten Handlungsmaxime erklärt hatte, keimte in Deutschland die Hoffnung auf eine neue Superdisziplin: Ethnologische Provenienzforschung. Projekte, vor allem Stellen für das akademische Prekariat, kündigten sich in großer Zahl am Horizont an. Nachhaltig, so tönte es, würde diese Entwicklung sein, denn in den Archiven der Museen schlummerten gewaltige Schätze. Einmal hätte die Ethnologie von einem Trend profitiert, den vor Jahren die in Deutschland traditionell mächtige Kunstgeschichte inauguriert hatte, und der in die skrupulöse Unterscheidung von Raub und Tausch, Kauf und Betrug, kurz: in Objektbiografien münden sollte. Aber ach, all dieses Unterscheiden und Zeigen, das Zeigen von Unterschieden in Ausstellungen, die Selbstproblematisierung des Museums im Museum, der Glaube an ein zunehmendes Wachstum nach innen wie nach außen, der die altehrwürdige Institution im Digitalzeitalter noch einmal hätte mobilisieren können, es war vergebens. Denn gleichsam im Vorgriff auf eine schwarz-grüne Koalition beeilten sich Kulturstaatsministerin Monika Grütters und ihre badenwürttembergische Kollegin mit einem Rückgabedekret. Weitere Amtskollegen folgten. Im Zweifel, so heißt es, sollten Museumsobjekte zurückgege- ben werden. Und zwar schnellstmöglich. Die dauertagende Wissenschaft hingegen klemmte den Schoßhundschwanz ein: nix zu machen, keine neuen Stellen, zumindest nicht über die knappe Befristung hinaus. Man hatte die Wissenschaft gerufen, dann brauchte man sie nicht mehr.

Schwalben sind Zugvögel, sie überwintern am Horn von Afrika. Anders als die deutsche Politik geraten sie moralisch nicht unter Zugzwang, und anders als viele Wissenschaftler ziehen sie einfach weiter, wenn es irgendwo zu kalt wird. Die Politik, aber auch die Presse, hat zuletzt nach dem von Erich Kästner prominent gemachten Motto agiert: Es gibt nichts Gutes, außer man tut es. Das ist ein Lyrismus, eine schöne Geste, und gesinnungsethisch ist daran zunächst auch wenig auszusetzen. Außer man führt ein anderes geflügeltes Wort ins Gefecht: Das Gegenteil von "gut" ist "gut gemeint". Und hier könnte die Wissenschaft - die Ethnologie, die Afrikawissenschaften, aber auch die Kunstgeschichte - durchaus korrigierend eingreifen, wenn sie es weniger auf das Nest abgesehen hätte, das ihnen von Zeit zu Zeit aus Mitteln von Bund und Ländern kredenzt wird. Denn:

1. Kategorien, die für die Raubkunstdebatte um die Enteignung jüdischer Kunstsammler gelten, lassen sich nicht ohne Weiteres auf Ethnografica übertragen, die in deutschen Museen ruhen, ganz egal ob in den Vitrinen oder Depots. Oder aber man weitet die Raub- 
kunstdebatte zur Museumsdebatte aus - dann wird es allerdings uferlos: Die Einrichtung der wichtigsten europäischen Museen ist maßgeblich durch die napoleonischen Kriegszüge bestimmt, schätzungsweise zu zwei Dritteln. Das heißt, ohne Raub gibt es keine Museen, nicht einmal die vornapoleonischen Wunderkammern reisender Adeliger. Es gibt darum überhaupt keinerlei unschuldige Museen, und selbst wenn man wie Präsident Macron Dependancen der eigenen Sammlungen im außereuropäischen Ausland anregt - allerdings eher in den Golfstaaten als in der sogenannten "Dritten Welt" -, wird man die Museen von ihrer Vergangenheit nicht reinigen können.

Die Raubkunstdebatte ging davon aus, dass Kunstschätze illegal erworben wurden. Ein großer Teil der an der Restitutionsdebatte Beteiligten will diesen Grundsatz ebenfalls anwenden. Sie vergessen dabei, dass (Gaben-)Tausch, Schenkungen etc., die bei vielen Objekten angegeben werden können, zwar auch in einem kolonialen Kontext vorkamen, aber doch tief eingebettet sind in menschliche Umgangspraktiken, dass also nicht einmal von einem kolonialistischen Kontext auf den Zwangscharakter der Gabe geschlossen werden kann. ${ }^{1}$ Zudem gibt es eben sehr viele Artefakte, zumal in deutschen Museen, die gar nicht erst aus deutschen Kolonien stammen. Wie H. Glenn Penny eindrucksvoll gezeigt hat, verdanken sie sich aber nicht selten einem kolonialen Kontext, an sich' - in dem Deutsche, wie Adolf H. Bastian, der Gründer des Berliner Völkerkundemuseums, als neutrale Mittler auftreten konnten. ${ }^{2}$ Aber wiederum gilt: die pauschale Verurteilung von Tausch als Raub wird der sozialen Natur des Menschen nicht gerecht.

2. Die Europäer haben im Nachgang der napoleonischen Raubzüge eine - nicht zuletzt von den Franzosen als den ersten Nutznießern - weitgehend widerspruchslos übernommene Kategorie der "Europäischen Kunst" oder „Europäi-

1 Der Ethnologe Fritz Kramer hat auf diese letztlich aus europäischem Exzeptionalismus herrührende Kritik hingewiesen: Ist der Fremde ein Mensch, in: Die ZEIT 20/2018. 2 Vgl. H. Glenn Penny, Im Schatten Humboldts. Eine tragische Geschichte der deutschen Ethnologie, aus dem Englischen von Martin Richter, München 2019: C. H. Beck. schen Kultur" entwickelt. Damit löste sich das Problem der Museen, die sich der Beraubung von Kollektiven verdankten. Erst die Enteignung von Individuen im Zuge der von Deutschland begonnenen Judenvernichtung hat den Begriff des Raubes wieder hervorgehoben. Die der Ermordung der europäischen Juden vorangegangene Enteignung war so betrachtet ein Akt wider die „Europäische Kunst", die von nun an mit jenem Blut überzogen ist, das ihre freie Zirkulation verhindert. Freie Zirkulation war schließlich das Kennzeichen europäischer Kunst - als jeder Kunst, die in Europa zirkulieren, gezeigt, besessen werden konnte. Genau genommen ist "Raubkunst" jene Kunst, die nicht mehr zirkulieren darf, die aus dem europäischen Geschehen genommen wird.

Eine Überlegung lautet deshalb, ob man nicht analog zum Begriff der „Europäischen Kunst" einen Begriff von "Weltkunst" etabliert, der eine der ganzen Menschheit gehörende Kunst bezeichnen und darum ihre freie Zirkulation, ihre Befreiung von partikularen kulturellen Identitätsansprüchen beanspruchen würde. Dies scheint aus mehreren Gründen schwierig. Zunächst, weil „europäische Kunst" durchaus einen partikularen Anspruch, nämlich einen kulturellen Hegemonieanspruch transportiert, dann, weil Menschheit als Kollektivsingular je von jemand ausgesagt wird (das alte Universalismusproblem), und schließlich, weil man tatsächlich keinen Gegenbegriff zu „Weltkunst" finden dürfte (außer: "Nichtkunst" oder "außerirdische Kunst"). Der Vernutzung von Artefakten zur Unterstreichung von Identitätsansprüchen lässt sich also scheinbar nicht durch deren Ausweitung vorbeugen.

3. Würde es also helfen, Identitätsansprüche einzugrenzen? Dazu müsste man sich noch einmal mit der Geschichte des "kulturellen Eigentums" auseinandersetzen, wie es die UNESCO nach dem Zweiten Weltkrieg entwickelt hat. ${ }^{3}$ Es steht zu erwarten, dass das entsprechende Konzept als eine Art Pufferung

3 Frühzeitig, in zunehmendem Maße seit den 1990er-Jahren, hat sich Kritik am angewandten Eigentumsbegriff formuliert, vgl. L. V. Prott, E. J. O'Keefe, ,Cultural Heritage' or ,Cultural Property'? In: International Journal of Cultural Property, 1/1992. Eine ideenhistorische Untersuchung scheint allerdings noch auszustehen. 
in der Auseinandersetzung des Kalten Krieges diente, wo es von den beiden Blockmächten jeweils in Anspruch gebracht werden konnte, um die Vereindeutigung ihrer Einflusssphären zu reduzieren. Ferner trug es zur Emanzipation ehemaliger Kolonialstaaten bei, die sich eben nicht nur als durch mehr oder weniger arbiträre Grenzziehungen und Roadmaps des Aufstieges ihrer gerade wichtigsten sozialen Klasse geformte ,imagined communities' (B. Anderson) definieren wollten, sondern ihre Daseinsberechtigung über ihre kulturelle Einheit zu bestimmen versuchten. Allerdings wird man einwenden müssen, dass gerade letztere in den meisten Fällen eine Fiktion darstellte. Nicht nur Eric J. E. Hobsbawm hat "invention of tradition" als Zusammenschluss von Praktiken aufgezeigt, in der sich z. B. das Britische Empire und die afrikanischen Nachfolgestaaten am wenigsten unterschieden.

"Kulturelles Eigentum" schließlich ist bisweilen selbst zum Synonym von Unterdrückung avanciert. Die Anmaßung von partikulärer kultureller Tradition als nationaler, die Weise, in der Minderheiten gleichzeitig konserviert, primitivisiert, ihrer politischen Rechte beraubt und dennoch als Aushängeschilder nationaler Kultur genutzt wurden, lässt sich vom kommunistischen Europa über Westafrika bis nach Südamerika, aber natürlich auch in heutigen südostasiatischen Staaten (in Vietnam die Jarai, in Thailand die Karen) aufzeigen. Für moderne Staaten, ob demokratisch, plebiszitär oder schlicht über eine Militärjunta und die Kraft des Stärkeren legitimiert, ist die identitätspolitische Ausbeutung von Minderheiten ebenso wie die Selbst-Indigenisierung ein vielfältig angewandtes (Stil-)Mittel. Zwar ließe sich denken, dass „kulturelles Eigentum" gerade der Auflösung in "nationales Eigentum" widerstünde - nur, Hand aufs Herz, wer außer die Nationalstaaten und ihre Allianzen, sollte dies durchsetzen? Ein UNESCO-Komitee? Mit der Legitimation durch wen? Die Source-Communities, sprich: jene Gemeinschaften, denen die Artefakte der Museen, falls überhaupt, im engeren Sinn gehören, weil sie in ihnen und für sie angefertigt wurden, ihren Sitz im jeweiligen sozialen Leben samt seiner Rituale und Gewohnheiten besaßen, sind eben entweder selbst über die Grenzen heutiger Nationalstaa- ten verstreut - dann ist es faktisch schwierig, ihnen etwas "zurückzugeben" - oder sie werden von jenen Gruppen beherrscht, die als Erben der Antikolonialbewegung heute antreten, das Label des Postkolonialismus kolonialistisch seinem Zweck zu entfremden. Schließlich: Wenn auf nationaler und regionaler Ebene gewählte deutsche Politiker über Rückgaben verhandeln, dann tun sie es mit national zuständigen Politikern - nicht mit Direktoren von Regionalmuseen oder gar Wissenschaftlern. Man sollte also nicht die Augen davor verschließen, dass "Rückgabe" jene Kaskade identitätspolitischer Ansprüche in Gang setzt, aus der im besten Fall harmlose Erfindungen, im schlimmsten allerdings Blutvergießen und Massaker hervorgehen.

4. Aber ist dieser Gedanke nicht selbst wiederum "kolonialistisch"? Der Einwand versteht sich in einem deutschen Text quasi als Reflex - gelegentlich als gesunder. Natürlich sollte man andere Staaten nicht bevormunden. Aber muss man innen deshalb Werkzeug und Material für ihre Unterdrückungsund Ausbeutungsanlagen zukommen lassen? Möglicherweise muss man das zulassen, weil es in der Logik nationalstaatlichen Handelns selbst liegt. Vielleicht gibt es eine Irreparabilität des Nationalstaates - in einem solchen Fall sollte man aber als Wissenschaftler nicht bloß verzweifeln, und wenn schon nicht gleich zum Anarchisten (in einer der societées contre l'etat, wie Pierre Clastres verlangte), so doch zum Bewahrer der wissenschaftlichen Autonomie werden. Denn zur Wissenschaft gehören die Ethnographica, weil sie eben nur im geringen Maß als Kunstwerke, vielmehr als Dokumente fremdkulturellen Lebens gesammelt und ausgestellt wurden.

Der Dokumentcharakter fordert die Besinnung der Wissenschaft, während das Rubrum des Kunstwerks die Zuständigkeit der Politik auf den Plan ruft. Dieser Punkt sollte bedacht und bei jeder Gelegenheit hervorgehoben werden. Dokument ist schließlich der am wenigsten eurozentrische Zugriff, er hält die Ethnographica in der Schwebe zwischen fremder Herkunft und eigener (europäischer) Aneignung. Die Rubrizierung als Kunst hingegen, die Ausdruck einer kulturellen Identität oder 
eines individuellen Genies sei (diese Kategorie wird seltener bemüht, auch weil sich die Herkünfte einzelner Gegenstände schwer eruieren lassen), erfolgt indes auf dem Boden einer mehr als zweitausendjährigen abendländischen Debatte, die mehr oder weniger teleologisch den Weg vom Kult zur als autonom verstandenen Kultur annimmt. Und nicht nur das: Sie kulminiert in der Vorstellung des bürgerlichen Subjekts als eben jenem, das die Trennung von Zweck- und Wertrationalität hervorgebracht hat, in der sich die Eigenlogik des Ästhetischen ausleben darf. Die Applikation des Kunstbegriffs auf ethnographische Artefakte ist also mindestens genauso ethnozentrisch wie soziozentrisch. Bevor unsere Kunstwissenschaft, unsere Museen nicht endlich so divers geworden sind, wie sie vorgeben - divers in Hinsicht auf Geschlecht, Ethnie, Klasse - und bevor nicht wenigstens einmal, wie es die Surrealisten forderten, die Kanalisation durch ihre heiligen Hallen geleitet worden ist, sollte man mit dem Kunstbegriff zurückhaltend umgehen. Oder ist etwa die fremdkulturelle Kunst das letzte Refugium der Kunst nach deren Ende geworden? Möglich, aber eben auch ein Kolonialismus unter anderen.

Die angeführten Punkte lassen sich verlängern, zudem lassen sie sich zu einer Reihe von Anklagen bündeln. Erhard Schüttpelz und Brigitta HauserSchäublin haben dies in einem sine ira, cum studio herausgegebenen Band Geschichts-Kultur durch Restitution? Ein Kunst-Historikerstreit getan, der schon als Preprint eine heftige Kritik im SPIEGEL provozierte. Ihre Mahnungen, nicht blindwütig der Ignoranz des „Gutes? Man tu' es!" zu folgen, verhallten erneut ungehört. Der Tenor der Rezension war, die Wissenschaftler mögen ja gerne streiten, nur sollte man bitte zeitgleich Schauräume und Depots leeren. Ein engerer Schulterschluss von Politik, Journalismus und Aktivismus ist kaum vorstellbar. Indirekt gaben die Journalisten damit einem der Hauptkritikpunkte von Schüttpelz recht: Es gehe um "Plünderung von Museen im Namen der historischen Gerechtigkeit", ausgehend von "Rechtsgrundlagen [...] im Widerspruch zu traditionellen Rechtsgrundsätzen." ${ }^{4}$ Die Möglichkeiten

4 Erhard Schüttpelz, Der kurze Moment der Restitutionsdebatte und seine Lange Dauer. Ein Zwillingstext, in: T. des Handelns müssen als ungemein beschränkt eingeschätzt werden, dass Handlungsoptionen im Feld der Restitution derartige Kräfte mobilisieren. Klimakrise, Corona-Pandemie - da steht man quasi ohnmächtig daneben, "Restitution nach Afrika" hingegen scheint noch einen Rest von Freiheitsgefühl zu verströmen.

Mit etwas Abstand zur Debatte sieht man allerdings, dass es die kritischen Stimmen der Mehrheit zu einfach gemacht haben. Anhand von Hauser-Schäublins im April in der NZZ erschienenem Beitrag über die Benin-Bronzen kann man dies illustrieren. ${ }^{5}$ Die Autorin, immerhin jahrzehntelang Direktorin des Zürcher Rietberg-Museums, setzte darin dem Narrativ von der britischen Strafexpedition, bei der die Werke gestohlen worden seien, das Bild des menschenopferhungrigen beninischen Königshofs entgegen. Dass afrikanische Potentaten nicht einfach Opfer waren, sondern schon früh ihre Kriege in Zulieferdienste für Sklaverei umwidmeten, ist bekannt; die Verve, mit der hier eine europäische Plünderung gegen notorische afrikanische ausgespielt wird, musste jedoch verstören. Denn was bedeutet es, wenn an den Bronzen, die für manchen Kunstliebhaber mit jenen von Riace zu vergleichen sind, Blut klebt? Bedeutet es, dass sie nun niemand gehörten? Sollte man sie den Opfern des längst untergegangenen Königreichs zurückgeben? Oder sollen sie in einem nigerianischen Nationalmuseum eine eigene Variante des Philhellenismus-Kultes eröffnen (Ich sah eine Auswahl im Stadtmuseum von Bologna, bewusst neben Werken der griechischen Antike platziert)? Norbert Elias, der 1962 im soeben dekolonialisierten Ghana erstmals Professor wurde, schrieb, er wolle nach Afrika gehen, um die Gründung der westlichen Zivilisation in situ zu verstehen. Civilization in a nutshell, the real Greek. Welcome to our welthistorisches Cinema. Immerhin: Bei allem Bohei um diese Edelplastiken, mit denen man so gerne das Humboldt-Forum unter dem Preußenkreuz eröffnen möchte: Ich kenne keinen Ethnologen, dem sie ernsthaft etwas bedeuteten.

Sandkühler, A. Epple, J. Zimmerer (Hg.): Geschichtskultur durch Restitution. Ein Kunst-Historikerstreit, Weimar: Böhlau 2021, hier S. 39.

5 Brigitta Hauser-Schäublin, Die lange Blutspur der BeninBronzen, in: NZZ am Sonntag, 25.04.2021. 
Und keinen Kunsthistoriker, der das Gegenteil behauptet.

Womöglich gibt man also auch gerne etwas zurück, weil es einem im Prinzip egal ist. Das wäre eine denkbar schlechte Grundlage, um mit der nichteuropäischen Welt das Gespräch zu vertiefen. Es wäre aber, was man in Deutschland sehr schätzt: nur recht und billig.

Andererseits geht es um weit mehr als um die Benin-Plastiken. Um Masken, für die man nicht genug tanzen kann, um Prachtboote, auf deren Stern die ganze Kosmologie der Südseebewohner dargestellt ist. Und natürlich um Objekte, die unauslöschbare Spuren in der westlichen Kulturgeschichte hinterlassen haben, etwa im Expressionismus. Der Ruf nach bedingungsloser Restitution verwandelte sich so gesehen rasch in einen Beitrag zur dauerhaften Entflechtung der deutschen Kulturgeschichte aus einer immer erst nachträglich zu rekonstruierenden Weltkulturgeschichte. Was bliebe dann übrig? Nationalstaatliche Säuberung und kontextlos Eigenes, das vermutlich nur neue Genie- und Heroengeschichten sprießen ließe, bis zur traurigen Ermattung. Während die Corona-Pandemie die Tücken der Globalisierung realistisch vor Augen führt, kann man deren unerwünschten Effekte durch Restitution wenigstens virtuell verdrängen. Aber glücklich wird man dabei nicht. Genauso wie die meisten Ethnologen wusste es Franz Kafka besser: „Wie kann man sich über die Welt freuen, außer wenn man zu ihr flüchtet?"6

6 Franz Kafka, Zürauer Aphorismen 1917 (A 118). 